Article

\title{
Investigating Commercial Filaments for 3D Printing of Stiff and Elastic Constructs with Ligament-Like Mechanics
}

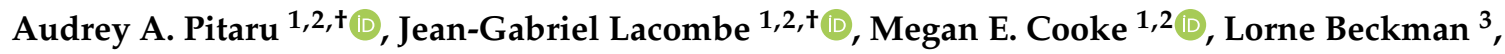 \\ Thomas Steffen ${ }^{3}$, Michael H. Weber ${ }^{1,2}$, Paul A. Martineau ${ }^{1,2}$ and Derek H. Rosenzweig ${ }^{1,2,4, * \text { (D) }}$ \\ 1 Division of Orthopaedic Surgery, McGill University, Montreal, QC H3A 1A1, Canada; \\ audrey.pitaru@mail.mcgill.ca (A.A.P.); jean-gabriel.lacombe@mail.mcgill.ca (J.-G.L.); \\ megan.cooke@mail.mcgill.ca (M.E.K.); Michael.weber@hotmail.com (M.H.W.); \\ paul.martineau@mcgill.ca (P.A.M.) \\ 2 Department of Experimental Surgery, McGill University, Montreal, QC H3A 1A1, Canada \\ 3 The Orthopaedics Research Lab, McGill University, Montreal, QC H3A 1A1, Canada; \\ lornebeckman@gmail.com (L.B.); tsteffen@orlmcgill.org (T.S.) \\ 4 Injury, Repair and Recovery Program, Research Institute of McGill University Health Centre, \\ Montreal, QC H3A 1A1, Canada \\ * Correspondence: derek.rosenzweig@mcgill.ca; Tel.: +01-514-934-1934 (ext. 43238) \\ + These authors contributed equally.
}

Received: 9 July 2020; Accepted: 10 September 2020; Published: 11 September 2020 updates

\begin{abstract}
The current gold standard technique for treatment of anterior cruciate ligament (ACL) injury is reconstruction with autograft. These treatments have a relatively high failure and re-tear rate. To overcome this, tissue engineering and additive manufacturing are being used to explore the potential of 3D scaffolds as autograft substitutes. However, mechanically optimal polymers for this have yet to be identified. Here, we use 3D printing technology and various materials with the aim of fabricating constructs better matching the mechanical properties of the native ACL. A fused deposition modeling (FDM) 3D printer was used to microfabricate dog bone-shaped specimens from six different polymers-PLA, PETG, Lay FOMM 60, NinjaFlex, NinjaFlex-SemiFlex, and FlexiFil—at three different raster angles. The tensile mechanical properties of these polymers were determined from stress-strain curves. Our results indicate that no single material came close enough to successfully match reported mechanical properties of the native ACL. However, PLA and PETG had similar ultimate tensile strengths. Lay FOMM 60 displayed a percentage strain at failure similar to reported values for native ACL. Furthermore, raster angle had a significant impact on some mechanical properties for all of the materials except for FlexiFil. We therefore conclude that while none of these materials alone is optimal for mimicking ACL mechanical properties, there may be potential for creating a 3D-printed composite constructs to match ACL mechanical properties. Further investigations involving co-printing of stiff and elastomeric materials must be explored.
\end{abstract}

Keywords: 3D printing; scaffolds; tissue engineering; elastic; mechanical strain; ligament; polymers

\section{Introduction}

The anterior cruciate ligament (ACL) is one of the most frequently injured structures during sporting or high impact activities [1]. It is one of two cruciate ligaments in the knee and ensures passive restraint of anterior translation and rotation of the tibia with respect to the femur, in part due to its anisotropic properties [2-4]. The ACL is composed of a highly organized collagen matrix consisting mainly of type I and III collagen, as well as elastin, proteoglycans, glycosaminoglycans, and adhesion 
proteins [5,6]. The ACL has been described as a continuum of fibres [7-9] or as three distinct fibre bundles [10-12]. In all cases, it is clear that the fibres of the ACL align in the same direction, parallel to the long axis of the ligament [8,13-15]. The aligned collagen fibres allow the ligament to withstand sizable forces with movement [16]. ACL injuries can result in reduced functional performance but are also associated with long-term clinical conditions that include meniscal tears, cartilage lesions and an increased risk of early onset post-traumatic osteoarthritis (OA) [17]. In traumatic joint injuries, the ACL tends to fail and tear until the ends are completely separated and the recovery process is slow due to its poor healing capacity often attributed to the avascular nature of the tissue $[14,18]$. Additionally, reattachment of torn ends has had limited clinical success [19]. The current gold standard of treatment for ACL injury is surgical reconstruction with autograft, most commonly of the patellar or hamstring tendons. However, autograft techniques are associated with donor site-related issues such as larger incisions and anterior knee pain [18]. Other drawbacks include the amount and availability of autograft tissues and complications related to graft harvesting [20]. Further, ligament does not fully recover following viscoelastic elongation [21]. Crawford et al. [22] performed a ten-year follow up for clinical outcomes after intra-articular non-artificial ACL reconstruction where they found that the overall cumulative ACL failure rate was $11.9 \%$. In addition, it was found that one in nine patients who undergo ACL reconstruction will have a re-rupture or clinical failure at long-term follow up.

Additive manufacturing, also known as three-dimensional (3D) printing, is a fabrication process by which 3D constructs are built in a layer-by-layer fashion using 3D computer-generated models. Additive manufacturing is open source and used for a variety of applications due to its ability to produce geometries and parts that are too complex for long-standing manufacturing processes that are subtractive in nature [23,24]. 3D printing of polymeric scaffolds can generate mechanically competent structures that can act as templates for tissue formation and regeneration $[25,26]$. 3D printing can also create sophisticated, porous scaffolds with complex geometry not possible with traditional manufacturing processes $[27,28]$. Using fused deposition modelling (FDM), many 3D printing parameters can be controlled. This enables a customizable approach to ensure that scaffold structural properties such as fibre orientation, pore size, and geometry can be optimized. A variety of biocompatible and resorbable materials have been used to generate ligament/tendon-like scaffolds such as poly-L-lactic acid, poly (urethaneurea) and polycarbonate poly (urethaneurea) [29]. However, there are very few studies into the use of 3D manufacturing and FDM for fabrication of scaffolds mimicking the biomechanical properties of the ACL.

To address shortcomings associated with surgical intervention and the poor healing of the ACL, researchers have explored tissue engineering (TE) and regenerative medicine strategies which aim to combine cells, scaffolds, and biologically active molecules [30]. 3D-printed scaffolds can be seeded with cells and then implanted into the injured site to allow for growth or regeneration of the tissue [28,31,32]. One concept is to use bioreactor-matured tissue engineered scaffolds to overcome the current limitations associated with surgical reconstruction. Furthermore, cell-seeded scaffolds form autografts that can overcome issues with supply shortage $[33,34]$. The implementation of tissue engineering harmonizes additive manufacturing and cell therapy to obtain state of the art tissue repair. However, it remains unclear as to whether 3D-printed polymers can be manufactured to match the biomechanical properties of the ACL.

In this study, low-cost FDM 3D printing was used to generate scaffolds from multiple materials with differing raster angles, and their mechanical properties were determined using tensile testing. The materials tested include polylactic acid (PLA), polyethylene terephthalate glycol (PETG), Lay FOMM 60, NinjaFlex, NinjaFlex-SemiFlex, and FlexiFil. PLA and PETG were selected due to their high ultimate tensile strengths (UTS). All other filaments were selected for their flexible nature. Although data on the stress properties are available for most of these materials in bulk, the effect of printing raster angle on their mechanical properties is unknown. Therefore, this study aims to fill this gap by testing not only different polymers, but also three differing raster angles to identify optimal combinations to match the mechanical strength of the ACL. 


\section{Materials and Methods}

\subsection{D Printing of Tensile Specimens}

An overview of the 3D printing and tensile testing process is shown in Figure 1. 3D printing was carried out with two FDM printers, the FlashForge Creator Pro (Flashforge; Los Angeles, CA, USA) and the Monoprice MP Select Mini v2 (Monoprice, Inc; Brea, CA, USA). Design and conversion processes were similar for both printers. SOLIDWORKS 2015 (Dassault Systèmes, SolidWorks Corporation, Waltham, MA, USA) was used to design a dog bone shape for tensile testing to induce fracture in the gauge section of the specimen. The CAD file was saved as a stereolithography (STL) file and sliced using Ultimaker Cura 4.3.0 (Ultimaker B.V.; Utrecht, Netherlands) software to obtain the G code for the Monoprice printer, and a x3g file extension for the FlashForge printer. The nozzle diameter for both printers was $0.3 \mathrm{~mm}$, the line width was $0.4 \mathrm{~mm}$, and printing was performed indoors in a temperature-controlled environment. The infill for all specimens was $100 \%$, with no distance between printed lines.

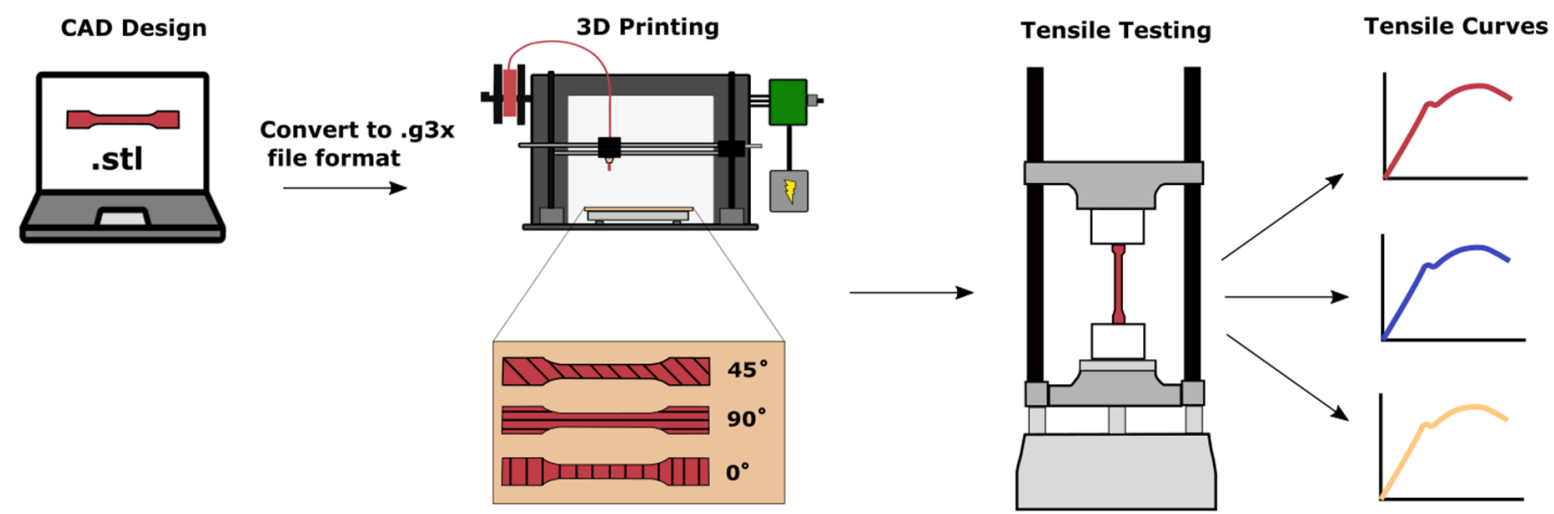

Figure 1. Schematic of overall procedure for 3D printing and mechanical testing of tensile specimens.

For each material, a total of 18 tensile specimens were produced. There were three different printing directions for each material, $0^{\circ}, 90^{\circ}$, and $45^{\circ}$ (see Figure 1 ). The $0^{\circ}$ raster angle was defined as horizontal filling along the width of the specimen, whereas the $90^{\circ}$ raster angle was printed longitudinally and the $45^{\circ}$ raster angle diagonally. For the $45^{\circ}$ specimens, the angle alternated between printed layers.

\subsection{Filaments}

The materials tested were: white PLA (MakerGeeks; Springfield, MO, USA); black PETG; (SUNLU; Commerce, CA, USA); white Lay FOMM 60 (MatterHackers; Burbank, CA, USA); blush NinjaFlex (Fenner Drives; Manheim, PA, USA); red NinjaFlex-SemiFlex (Fenner Drives; Manheim, PA, USA); and blue FlexiFil (Formfutura; Nijmegen, Netherlands). PLA and PETG were selected due to their high UTS, since the ACL is a load-bearing band in the knee. NinjaFlex is a thermoplastic polyurethane composition that allows for $660 \%$ elongation. NinjaFlex-SemiFlex is made of the same polymers, but is formulated to be slightly more rigid, to allow for an increased UTS. FlexiFil is a rubberized thermoplastic co-polyester. These three filaments were selected due to their flexible nature, in an attempt to mimic the viscoelastic nature of the native ACL. Lay FOMM 60 is a highly porous material composed of a flexible thermoplastic polyurethane (TPU) and polyvinyl alcohol (PVA) blend. The PVA component is water-soluble and can be washed out to create flexible, nanoporous, sponge-like structures following printing [35]. The manufacturer-reported mechanical properties for the used materials can be found in Table 1. Lay FOMM 60 did not have any reported mechanical properties from the manufacturer. 
Table 1. Reported tensile values for raw filaments.

\begin{tabular}{ccccc}
\hline Material & $\begin{array}{c}\text { Yield Tensile } \\
\text { Strength (MPa) }\end{array}$ & $\begin{array}{c}\text { Ultimate Tensile } \\
\text { Strength (MPa) }\end{array}$ & $\begin{array}{c}\text { Tensile Modulus } \\
\text { (MPa) }\end{array}$ & $\begin{array}{c}\text { Manufacturer } \\
\text { Datasheet }\end{array}$ \\
\hline NinjaFlex & 4 & 26 & 12 & {$[36]$} \\
SemiFlex & 9 & 43 & 25 & {$[37]$} \\
FlexiFil & & 24 & 95 & {$[38]$} \\
PLA & 35.9 & 26.4 & 2300 & {$[39]$} \\
PETG & 53 & & 2100 & {$[40]$} \\
\hline
\end{tabular}

The Lay FOMM 60 filament was heated in an oven for three hours at $80^{\circ} \mathrm{C}$ before printing to remove all moisture. The printed specimens were rinsed for three days in double distilled water to wash out most of the rigid PVA, replacing the water each day.

The printing parameters used for each material can be found in Table 2. For PLA, layer height was set to $0.2 \mathrm{~mm}$, flow rate was set to $100 \%$, retraction was enabled, and the printing speed was offset between $65 \%$ and $90 \%$ based on the appearance of the tensile specimen. Similar settings were applied to PETG but a skirt and raft were used to enhance build plate adhesion, and the print speed was set to $100 \%$. As for NinjaFlex-SemiFlex, referred to here as SemiFlex, initial layer height was set to $0.3 \mathrm{~mm}$, flow rate was set to $102 \%$ offset, retraction and combing were enabled, and the printing speed was set between $55 \%$ and $65 \%$ offset. The same settings were applied to FlexiFil, but the print speed was set to $100 \%$. The NinjaFlex filament had an initial layer height of $0.3 \mathrm{~mm}$, a skirt was used, the flow rate was set to $110 \%$, and retraction was disabled. Finally, Lay FOMM 60 was printed with initial layer height of $0.2 \mathrm{~mm}$ using a skirt, the flow rate set to $100 \%$, retraction enabled, and a printing speed set between $40 \%$ and $95 \%$ offset.

Table 2. Printing parameters of all materials.

\begin{tabular}{ccccc}
\hline Material & $\begin{array}{c}\text { Nozzle } \\
\text { Temperature }\left({ }^{\circ} \mathbf{C}\right)\end{array}$ & Bed Temperature $\left({ }^{\circ} \mathbf{C}\right)$ & $\begin{array}{c}\text { Initial Layer } \\
\text { Speed (mm/s) }\end{array}$ & Speed $(\mathbf{m m} / \mathbf{s})$ \\
\hline PLA & 205 & 70 & 17.5 & 35 \\
PETG & 240 & 80 & 17.5 & 35 \\
SemiFlex & 215 & 50 & 15 & 30 \\
FlexiFil & 215 & 55 & 15 & 30 \\
NinjaFlex & $230-240$ & 55 & 10 & 15 \\
Lay FOMM 60 & $220-225$ & 50 & 15 & 25 \\
\hline
\end{tabular}

\subsection{Light Microscopy}

The microscopic images of the printed constructs were captured with a Leica MS5 stereomicroscope, using the 1.6 magnification lens adapter for iPhone. Sample light microscopy images of the three raster angles are shown in Figure 2.
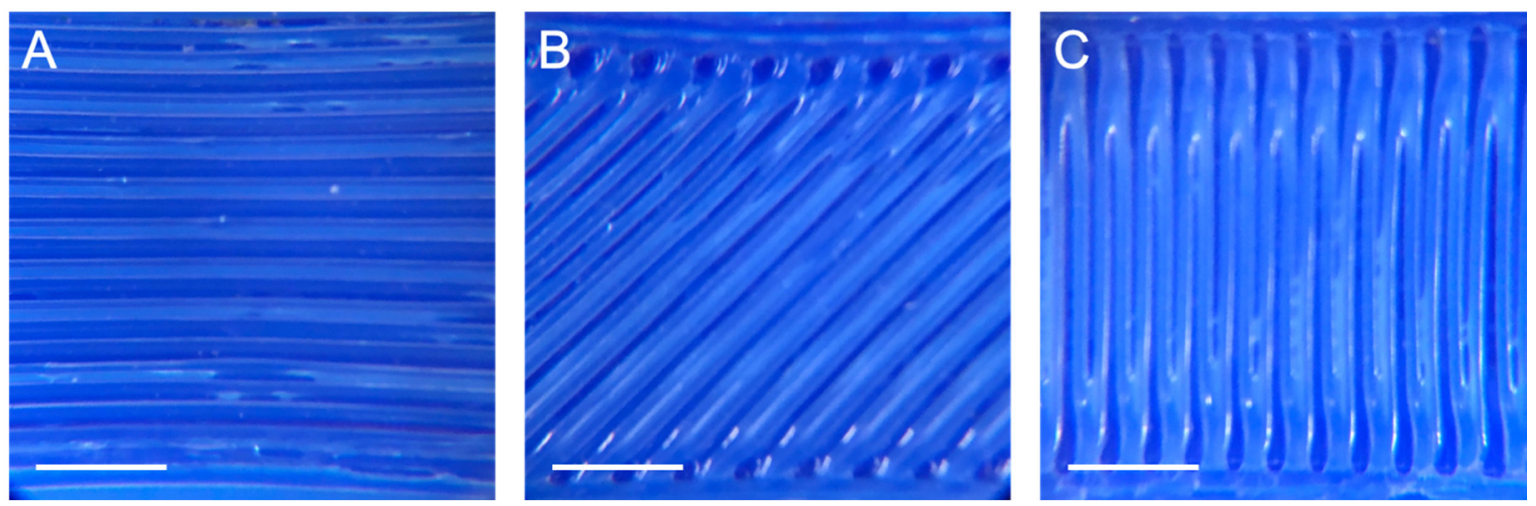

Figure 2. Light microscopy images of FlexiFil printed with raster angles of (A) $90^{\circ},(\mathbf{B}) 45^{\circ}$ and (C) $0^{\circ}$. Scale bar $=2.5 \mathrm{~mm}$. 


\subsection{Mechanical Testing}

Tensile testing was performed using the Mini-Bionix 858 (MTS; 14,000 Technology Dr. Eden Prairie, MN, USA). All specimens were tested with the TestStar II (MTS; 14,000 Technology Dr. Eden Prairie, MN, USA) software. A large, built-in MTS, Model \#662.20D-03 load cell was used in the calibrated 2000 N range. A smaller Model LCCD-100 (Omegadyne; 800 Connecticut Ave. Suite 5N01, Norwalk, CT 08654, USA) load cell with a capacity of $444 \mathrm{~N}$ was used for the Lay FOMM 60 specimens. The force and displacement data were recorded at $10 \mathrm{~Hz}$. The displacement was set to a maximum of $95 \mathrm{~mm}$. The test speed was $0.3 \mathrm{~mm} /$ second corresponding to $1 \%$ strain/second based on a nominal 30 $\mathrm{mm}$ gauge length. All tensile tests were performed at ambient room temperature. Lay FOMM 60 was stored in water until three hours before testing. The gauge, width, and thickness of the gauge were measured using a caliper.

\subsection{Tensile Analysis}

All force and displacement data were converted to stress and strain data using the specimens' cross-sectional areas and initial gauge lengths. Stress and strain were calculated by Equations (1) and (2), respectively.

$$
\begin{gathered}
\text { Stress }(\mathrm{Pa})=\frac{\text { Force }(\mathrm{N})}{\text { Area }\left(\mathrm{mm}^{2}\right)} \\
\text { Strain }(\%)=\frac{\text { Displacement }(\mathrm{mm})}{\text { Original length }(\mathrm{mm})}
\end{gathered}
$$

To ensure the replicability of analyses, force and displacement data were zeroed at the same points for all materials prior to conversion to stress and strain data. The stress-strain curves of PLA and PETG were used to calculate the Young's modulus, UTS and percent strain at failure. The Young's modulus for PLA and PETG was calculated as the ratio of tensile stress to tensile strain in the linear portion of each stress-strain curve (Equation (3)). UTS and percent strain at failure were observed directly from the stress-strain curve.

$$
\text { Young's and Apparent Modulus }=\frac{\text { Stress }}{\text { Strain }}
$$

Conventional UTS, percent strain at failure and yield strength values were not used to characterize the flexible materials due to atypical failures (occurring past 100\% strain). An apparent Young's modulus and atypical yield point were defined to assess the change in tensile elongation behavior as the flexible specimens are stretched past their elastic limit. The apparent Young's modulus used to describe flexible materials was obtained by determining the ratio of tensile stress to tensile strain (Equation (3)) in a restricted region of interest between strains of 0 and 5\%. This region of interest limits the analysis to a semi-linear curve that approximates the stiffness of flexible polymers and acts as a more accurate benchmark for elasticity analysis since a conventional Young's modulus cannot be used. The flexible yield point was defined as the stress and strain values at which a $2 \%$ offset line parallel to the semi-elastic slope of the stress-strain curve intersects the non-linear portion of the curve. The flexible yield point was used to approximate the point at which flexible materials start to experience plastic deformation. The stress-strain curves of flexible materials were used to determine additional properties of stress at $5 \%$ strain and stress at $20 \%$ strain.

\subsection{Verification of Gauge Displacement}

Due to the elastic nature of a number of tested materials, the gauge elongation was verified to ensure that the grip section elongation was negligible. The open-source Tracker video analysis and modeling tool was used to assess the movement of two points at opposite ends of the gauge 
section. Videos were recorded using an iPhone camera and uploaded to the software for analysis. Three independent Lay FOMM 60 tensile tests for each raster angle were performed.

\subsection{Statistical Analyses}

At least five independent tensile tests for each material and each raster angle were used to conduct the statistical tests, with the exceptions of PLA $0^{\circ}$ and PETG $0^{\circ}$ raster angles which each had 3 independent tests. Statistical analyses of stiff and flexible materials were separated into 2 groups due to their mechanics; comparisons were made within and not across groups. Statistical analyses of stiff materials, PLA and PETG, were performed comparing UTS, Young's modulus, and percent strain at failure within raster angles using unpaired $t$ tests. Statistical analyses for flexible materials for each raster angle were also performed comparing their apparent modulus and the stress at $5 \%$ and $20 \%$ strain using an ordinary one-way analysis of variance (ANOVA). Tukey HSD post-hoc tests were performed for comparisons between materials with the same raster angle, as well as between the three raster angles for each material. $p<0.05$ was considered statistically significant. Analyses were performed using GraphPad Prism 6.0 (GraphPad Software, La Jolla, CA, USA).

\section{Results}

\section{Tensile Properties}

PETG and PLA showed traditional deformation profiles, while the other materials showed more elastomeric behavior. For this reason, they were split into two groups for analysis: stiff (PLA and PETG) or flexible (Lay FOMM, SemiFlex, NinjaFlex and FlexiFil). The specimens were designed to fail in the gauge region. However, four PLA $-0^{\circ}$ specimens failed above the gauge region. This could be due to a defect in the specimen; consequently, all four specimens were excluded from the data. Most of the flexible materials did not reach failure as displacement was limited to $90 \mathrm{~mm}$ by the Mini-Bionix testing machine. The only flexible materials that failed were FlexiFil and Lay FOMM 60, both at $0^{\circ}$ and $45^{\circ}$ raster angles. For Lay FOMM $60-0^{\circ}$ samples, the outline of the specimen did not adhere well to the infill, which caused the shape of the specimen to warp. To overcome this, the excess filament of the outline was trimmed off using scissors prior to testing.

Stress-strain curves for all materials are shown in Figure 3. Individual curves were used to determine the UTS, Young's modulus, and the strain percentage at failure for the stiff specimens, and apparent modulus and the stress at $5 \%$ and $20 \%$ strain for the flexible specimens. For both PLA and PETG, clear regions of linear behavior are seen in the low strain percentage regions (at all raster angles). When considering the flexible materials, the stress-strain behavior of these polymers does not follow the typical tensile curve with an elastic region followed by a plastic region and in most cases, such as FlexiFil- $90^{\circ}$, is more akin to elastomeric deformation. The linear portion commonly seen in low-strain regions was not distinct for NinjaFlex or Lay FOMM 60.

The calculated UTS, Young's Modulus and percentage strain at failure for stiff materials are shown in Figure 4; corresponding results of one-way ANOVA of the effect of raster angle are shown in Table 3. In the case of PETG, raster angle had a significant effect on UTS and Young's modulus as shown in Figure 4 A,B, respectively. The strain at failure for PETG was not significantly different. As for PLA specimens, Table 3 shows that there were statistically significant differences for UTS and strain at failure, but no significant difference for the Young's modulus. PLA was unique in that the trend of UTS and percentage strain at failure was opposite to all other materials, with $0^{\circ}$ having significantly higher values than $90^{\circ}$. In terms of the comparison between materials, Figure 4A,B demonstrate statistically significant differences for UTS and Young's modulus between PLA and PETG specimens at each raster angle. As for percent strain at failure (Figure 4C), only at $90^{\circ}$ were PLA and PETG significantly different $(p=0.0317)$.

Figure 5 shows calculated apparent modulus (Figure 5A), stress at 5\% strain (Figure 5B) and stress at 20\% (Figure 5C) strain for flexible materials; corresponding results of one-way ANOVA of 
the effect of raster angle are shown in Table 4. Raster angle caused a statistically significant difference in apparent moduli of Lay FOMM 60, SemiFlex, and NinjaFlex (Table 4). Further, apparent moduli were significantly different between raster angles of $0^{\circ}$ and $90^{\circ}$ in these three materials. There were no significant differences in apparent moduli either between angles or within the three angles for FlexiFil. There are statistically significant differences between raster angles in terms of stress at $5 \%$ strain (Figure 5B) only for Lay FOMM 60. A difference exists between both $0^{\circ}$ and $90^{\circ}(p=0.0031)$, and $45^{\circ}$ and $90^{\circ}(p=0.0011)$ raster angles. No significant differences were found for SemiFlex, FlexiFil and NinjaFlex. The stresses at $20 \%$ strain were statistically significant within all flexible materials, as can be seen in Figure 5C. Lay FOMM 60 was the only flexible material in which apparent moduli and the stress at $5 \%$ and $20 \%$ strain were significantly different within the material for all three raster angles (Table 4).

Table 3. Results of one-way ANOVA comparing raster angles on mechanical properties of PLA and PETG.

\begin{tabular}{cccc}
\hline \multirow{2}{*}{ Material } & \multicolumn{3}{c}{ Effect of Raster Angle on Mechanical Properties } \\
\cline { 2 - 4 } & UTS & Young's Modulus & Strain at Failure \\
\hline PLA & 0.0300 & 0.6114 & 0.0280 \\
PETG & 0.0232 & 0.0053 & 0.9606 \\
\hline
\end{tabular}
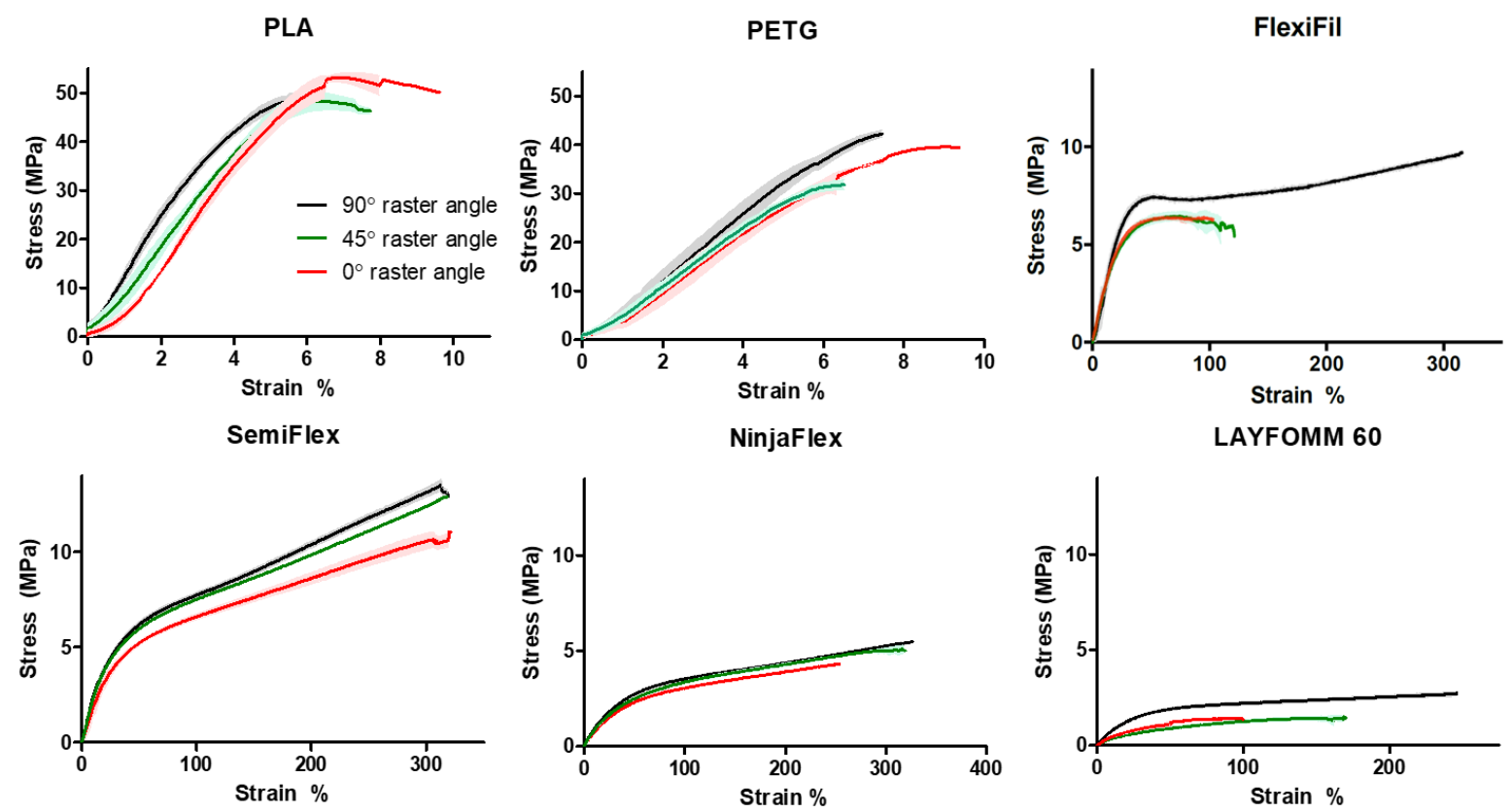

Figure 3. Stress-strain curves for all materials $(n=3)$ at each raster angle. Shaded zones around plot lines indicate the mean \pm SD. In some samples, error bars $(\mathrm{SD})$ are too small to be displayed.

A comparison of the significance values for the apparent modulus and the stress at 5\%,20\%,50\% and $100 \%$ strain for the flexible specimens for the $0^{\circ}, 45^{\circ}$, and $90^{\circ}$ raster angle can be found in the supplementary data (Tables S1-S3). Significant differences were apparent for most of the material comparisons at $5 \%$ strain for raster angle $0^{\circ}$ (Table S1). At strains of $20 \%$ and $50 \%$ for the same raster angle, significant differences were found for all materials. Data for stress at $100 \%$ strain for Lay FOMM are not available since the specimens failed before reaching the latter strain. At raster angles of $45^{\circ}$ and $90^{\circ}$, most comparisons were significantly different. Overall, Lay FOMM 60 and NinjaFlex were the only materials that were consistently not statistically different.

The experimental findings were then compared to previously published values of ACL mechanical properties, using a Young's modulus value of $278 \mathrm{MPa}$ [41], UTS of $35 \mathrm{MPa}$ [41], and strain at failure of 
28\% [42]. More specifically, these data were compared to the mechanical properties of all six materials with the ACL data normalized to 100\% (shown graphically in Figure 6, mean values are presented in Table 5). The Young's modulus values of PLA and PETG were around 2.5 and 4-fold greater than the ACL. As for UTS, the PETG specimens at $0^{\circ}$ and $45^{\circ}$ were very close in value to the UTS of the ACL, whereas the PLA specimens were slightly higher. The stiff materials did, however, have strain at failure values that were much lower the ACL. Opposite trends were seen for the flexible materials. The apparent moduli and flexible yield point were much lower than the native ACL's Young's modulus and the strain at flexible yield was higher than that of the ACL for all flexible materials at all raster angles. FlexiFil, SemiFlex and Lay FOMM had strain at flexible yield values around 1.5-fold higher than the native ACL, but NinjaFlex was 2-fold higher.
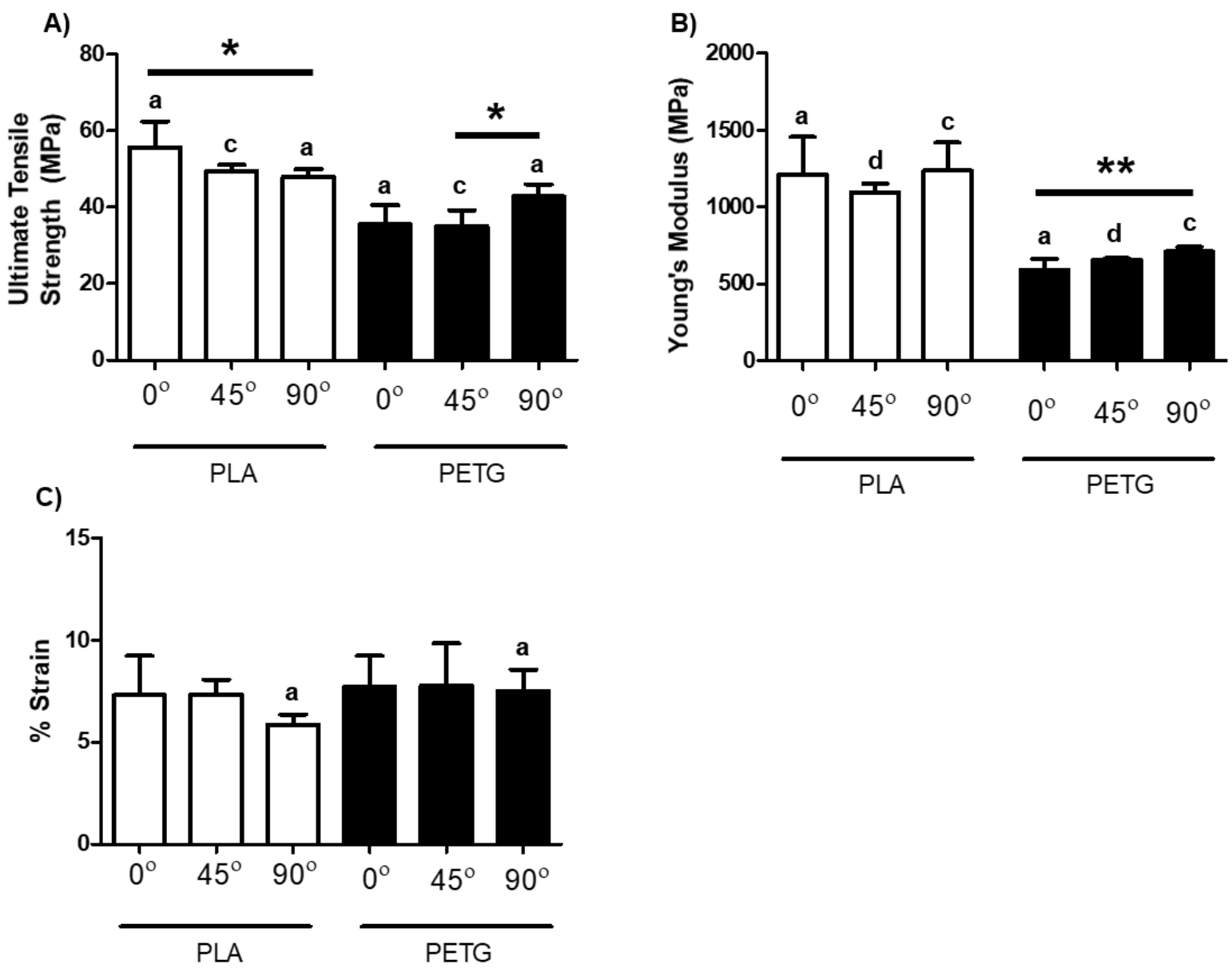

Figure 4. Mechanical properties of PLA and PETG at three differing raster angles: $0^{\circ}, 45^{\circ}$, and $90^{\circ}$. (A) UTS; (B) Young's modulus; (C) percent strain at failure. The letter denotation indicates a significant difference exists between PLA and PETG within the same raster angle. ${ }^{\text {a }}$ Significant difference of $p<0.05 .{ }^{\mathrm{b}}$ Significant difference of $p<0.01$. ${ }^{\mathrm{c}}$ Significant difference of $p<0.001$. ${ }^{\mathrm{d}}$ Significant difference of $p<0.0001$. ${ }^{*} p<0.05$. ${ }^{* *} p<0.01$. Data shown represent the mean $\pm \mathrm{SD}$. ${ }^{\mathrm{a}-\mathrm{d}}$ indicates significant differences comparing the same raster angle between different materials, while * indicates significant differences between raster angles of the same material. 
(A)

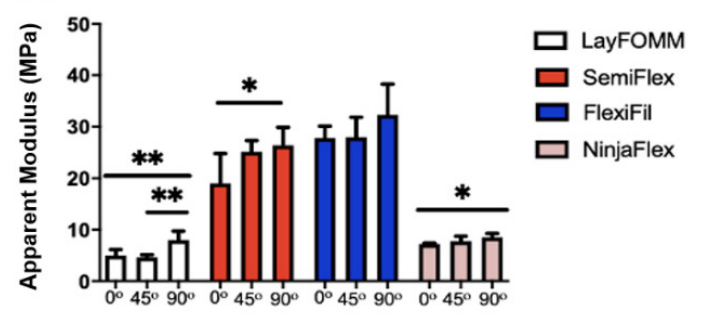

(B)

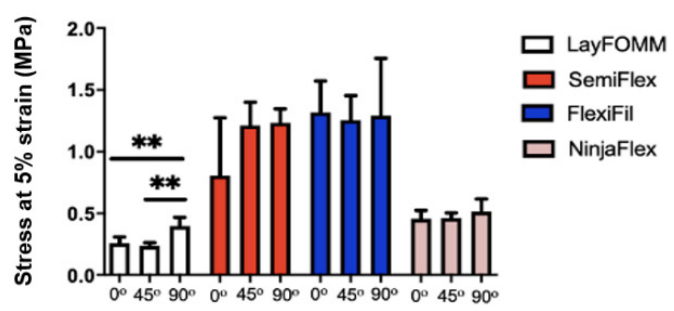

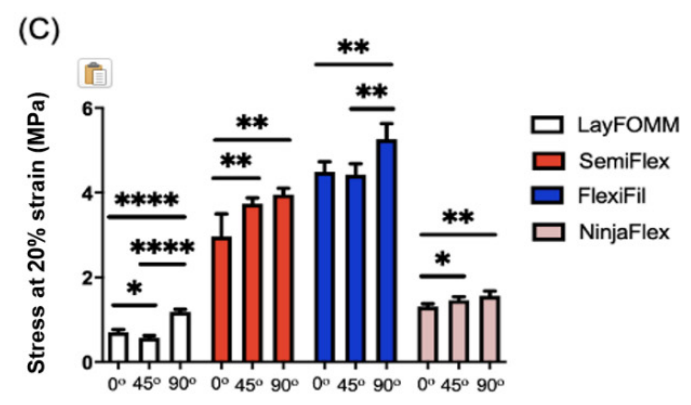

Figure 5. Mechanical properties of the Lay FOMM 60, SemiFlex, FlexiFil, and NinjaFlex materials for three raster angles: $0^{\circ}, 45^{\circ}$, and $90^{\circ}$. The mechanical properties displayed are: (A) apparent modulus; (B) stress at $5 \%$ strain; (C) stress at $20 \%$ strain. ${ }^{*} p<0.05,{ }^{* *} p<0.01,{ }^{* * * *} p<0.0001$. Data shown represent the mean $\pm \mathrm{SD}$.

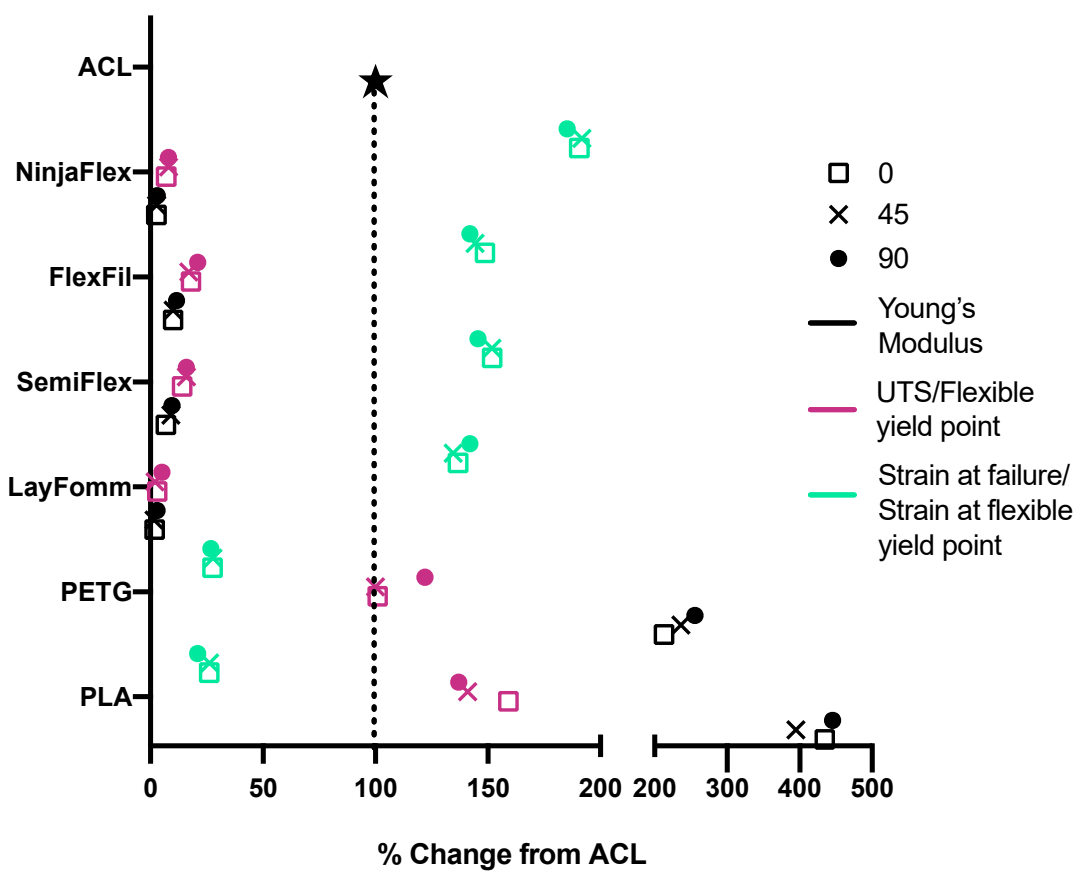

Figure 6. Percentage change of values compared to published native ACL mechanical properties. Raster angles are represented as $0^{\circ}$ - square, $45^{\circ}$ - cross, and $90^{\circ}$ —circle. Young's (stiff) or apparent (flexible) modulus values are shown in black, UTS (stiff) or flexible yield point (flexible) are pink and strain at either failure (stiff) or flexible yield point (flexible) are green. Mean values are plotted. 
Table 4. Results of one-way ANOVA comparing effects of raster angle on mechanical properties of flexible materials.

\begin{tabular}{cccccc}
\hline \multirow{2}{*}{ Material } & \multicolumn{5}{c}{ Effect of Raster Angle on Mechanical Properties } \\
\cline { 2 - 5 } & Apparent Modulus & $\mathbf{5 \%}$ Strain & $\mathbf{2 0 \%}$ Strain & $\mathbf{5 0 \%}$ Strain & $\mathbf{1 0 0 \%}$ Strain \\
\hline Lay FOMM 60 & 0.0018 & 0.0008 & $<0.0001$ & 0.0193 & N/A \\
SemiFlex & 0.0323 & 0.0746 & 0.0012 & 0.0006 & 0.0003 \\
FlexiFil & 0.2166 & 0.8273 & 0.0012 & 0.0119 & 0.0471 \\
NinjaFlex & 0.0489 & 0.4141 & 0.0028 & 0.0019 & $<0.0001$ \\
\hline
\end{tabular}

Table 5. Mechanical properties of all specimens and ACL literature values.

\begin{tabular}{ccccc}
\hline \multirow{2}{*}{ Material } & \multicolumn{3}{c}{ Mechanical Properties } \\
\cline { 2 - 5 } & Raster Angle $\mathbf{~}^{\circ}$ ) & $\begin{array}{c}\text { Young's Modulus } \\
\text { (MPa) }\end{array}$ & $\begin{array}{c}\text { UTS/Flexible } \\
\text { Yield Point (MPa) }\end{array}$ & $\begin{array}{c}\text { Strain at Failure/Strain at } \\
\text { Flexible Yield (\%) }\end{array}$ \\
\hline \multirow{2}{*}{ PLA } & 0 & 1208 & 55.72 & 7.301 \\
& 45 & 1098 & 49.43 & 7.347 \\
PETG & 90 & 1238 & 47.98 & 5.857 \\
& 0 & 591.6 & 35.50 & 7.731 \\
Lay FOMM 60 & 45 & 656.3 & 34.96 & 7.786 \\
& 90 & 710.7 & 42.85 & 7.509 \\
SemiFlex & 0 & 5.040 & 1.017 & 38.27 \\
& 45 & 4.622 & 0.8152 & 37.69 \\
FlexiFil & 90 & 18.992 & 1.711 & 39.76 \\
& 45 & 25.16 & 4.822 & 42.53 \\
NinjaFlex & 90 & 26.39 & 5.605 & 42.47 \\
& 0 & 27.82 & 5.687 & 40.75 \\
ACL & 45 & 27.96 & 6.129 & 41.63 \\
& 90 & 32.30 & 5.992 & 40.39 \\
& 0 & 7.244 & 7.189 & 39.75 \\
& 45 & 7.768 & 2.404 & 53.38 \\
& 90 & 8.505 & 2.650 & 53.70 \\
& - & $278[40]$ & 2.797 & 51.85 \\
& & & $35[40]$ & $28[41]$ \\
\hline
\end{tabular}

\section{Discussion}

ACL tears are very common; the current standards for surgical treatment are not as strong as the original ligament and have relatively high failure and re-tear rates. More effective ligament reconstruction strategies are therefore necessary. The fabrication of scaffolds for tendon and ligament tissue engineering has utilized numerous synthetic biomaterials, such as polycaprolactone, polyglycolic acid, poly(lactic-co-glycolic acid), poly-L-lactide, and polyurethane urea, as well as other techniques: electrospinning, knitting, melt extrusion-based 3D-bioplotting, and 3D braiding [13,30]. The technique of FDM in particular has been used to print different polymers to determine their tensile properties [43]. However, no studies to our knowledge have investigated raster angle in FDM printing to optimize biomaterial mechanics for ACL reconstruction. The strategy used for 3D printing in this study was a good fit. We produced tensile specimens that had uniform surface appearance, despite the use of different materials with varying properties. Tensile testing was then performed to determine the effect of changing raster angle on their appropriateness for use as mechanically functional ligament replacements.

Values for the mechanical properties of the ACL are inconsistent in the literature. It is difficult to assess the cross-sectional area of the ACL and to define an appropriate gauge length, which are needed to calculate the stress and strain. Thus, tensile properties of the ACL are often described in terms of force and elongation [44]. However, these properties are influenced by the specific geometry of each construct, which means they cannot be compared to anything other than ACL data. For the purposes of this study, stress and strain were chosen as the properties to be calculated since they allow for the 
possibility of comparison between the ACL and other materials, despite the fact that limited data on stress and strain for the ACL are available. It is also important to note the rationale behind selecting certain values for the mechanical properties. There is no literature on 3D printing of elastomers to mimic ligaments and therefore no data for direct comparison. The stress at 5\% strain was selected as peak strains produced during activities of daily living have been found to be close to $4 \%$ for the ACL [45]. The maximum strain for the ACL has been reported between $14.0 \%$ and 14.4\% [46] in some studies, and close to $19.1 \%$ in others [47]. A $20 \%$ strain was selected as it is above the values reported. It is important to note that stress at $100 \%$ and $300 \%$ strains is common for stress-strain curve analysis of elastomers. Despite being of limited relevance for the study of the ACL, stresses at $100 \%$ strain were still included to further characterize the materials which may be applicable in other, more flexible ligaments or tissues.

$3 \mathrm{D}$ printing is an established technique in a number of industries, and it is interesting to explore the feasibility of 3D-printed thermoplastics for ACL substitutes. PETG showed UTS values that were very close to those of the ACL (37.77 vs. $35 \mathrm{MPa}$ ). The Young's modulus was higher than that of the ACL (652.87 vs. $278 \mathrm{MPa}$ ) and the strain at failure was lower (7.68 vs. $28 \%$ ). These tensile values do not allow for a satisfactory factor of safety and therefore signifies that PETG scaffolds printed using the stated parameters may not be viable mechanical substitutes for the ACL despite presenting similar tensile properties. PLA constructs presented Young's modulus (1181 MPa) and UTS (51.04 MPa) values which were above those of the ACL. However, its strain at failure is lower than ACL values found in literature (6.835 vs. $28 \%$ ). The apparent moduli, stress at $5 \%$ and $20 \%$ strains, and the flexible yield point of all flexible materials was significantly lower than that of the native tissue. Only the strain at flexible yield values for all flexible materials were larger than the ACL's strain at failure. Because the Young's modulus and UTS of stiff materials are higher than that of the ACL, it may be possible to co-print them with a flexible material in order to increase the strain at failure and produce a composite scaffold with tensile properties very close to that of the ACL. Lay FOMM 60 is a good candidate for co-printing with a stiff material. In the removal of PVA with washing in water, micropores are revealed in the Lay FOMM surface. Numerous studies have shown the importance of porosity in scaffolds for cell attachment and proliferation [28,48] and the use of Lay FOMM 60 with PLA or PETG may enhance such properties. Our group has already reported that Lay FOMM 60 shows good cell viability in vitro and can deliver chemotherapeutics [35,49]. Lay FOMM's ability to deliver small molecules may enable the delivery of growth factors or hormones to enhance proliferation and matrix formation of ligament fibroblasts.

The impact of raster angle on the tensile properties was also investigated. It has been shown that aligned fibres result in higher tensile properties in scaffolds [50], and that increased fibre alignment leads to increased tissue stiffness [51,52]. Fibres must be printed in all orientations to replicate the anisotropic properties of the native ACL. A strong decline in tensile properties between raster angles may affect the mechanical stability of the graft in tension and cause tensile failure. It is important to consider the impact of raster angle since the graft needs to perform in many tensile planes at once. Additionally, combining the flexible and stiff materials may better mimic the anisotropic properties of the ACL, which has not been accomplished thus far to our knowledge. Raster angle had a significant impact on UTS and Young's modulus of the PETG specimens and on the UTS and strain at failure of the PLA specimens. This indicates that the tensile properties of PETG and PLA constructs fabricated using the stated parameters can be altered by their fibre orientation. Such alterations may be beneficial in replicating the ACL's anisotropic behavior. Flexible materials were all significantly impacted by the raster angle, except for FlexiFil. The raster angle had a significant effect on all mechanical properties for Lay FOMM 60 and all mechanical properties except for 5\% strain for NinjaFlex and SemiFlex. As for FlexiFil, the only mechanical properties that were impacted by the raster angle were the strains at $20 \%, 50 \%$ and $100 \%$, whereas the apparent modulus and 5\% strain were not statistically significant. Apparent modulus and 5\% strain are very important parameters regarding the mechanical properties of the ACL, so changing raster angle can be considered not important for FlexiFil. In conclusion, in terms 
of raster angle, Lay FOMM 60, SemiFlex, and NinjaFlex may be used to replicate the anisotropic properties of the ACL and may be more appropriate flexible co-printing candidates.

Use of FDM for fabrication of ACL scaffolds presents limitations. First, materials that possess appropriate properties for FDM such as viscoelasticity, thermoplasticity, and melting/solidification are limited. Further, the use of commercially sourced materials means that their exact composition is often proprietary (as in this study). Scaffold design is also restricted since the viscosity of the molten polymers only allow for the fabrication of structures with a bottom-up design approach. A limitation of the current study was the lack of testing to ensure that there was not under-extrusion of the interior of the specimen. Future studies should include precision weighing of the specimens in order to prevent this issue. If under-extrusion is found, the specimens should then be reprinted since mechanical stability is imperative in the ACL and previous studies have indicated a correlation between polymer weight and mechanical properties [53]. According to Perego et al. [54], the tensile strength was affected by the molecular weight of the specimen, whereby a larger molecular weight resulted in a higher tensile strength. Furthermore, Wittbrodt and Pearce [55] found that colouring agents altered the percent crystallinity, which had an impact on tensile strength. Wimpenny et al. [56], however, stated that colour of the filament has little effect on the tensile strength of FDM constructs. As no consensus has been reached in the literature, the colour of the filament was not explored in this study. It is known that the crystallinity impacts the tensile strength [55,57], therefore future studies should evaluate percent crystallinity of the material, weigh the specimens, and use uncoloured, natural filament to prevent confounding contributions of colourants and their sources. Another source of uncertainty is torsional forces. Although we investigated the tensile properties of polymeric materials, the response to torsional stresses and strains is unknown. It is believed that the raster angle of 3D-printed constructs may have an impact on torsional stress distribution within the material. Due to the ACL's multi-axial range of motion, it is important to also investigate these properties to ensure the mechanical accuracy of the scaffolds. Such torsional stresses could not be investigated in this study but should be considered in future investigations. Additionally, the stress-strain curves shown in this study assume a fixed cross-sectional area, which is not accurate for elastomeric specimens during elongation.

The ACL mechanical properties were selected from specific publications as a point of reference [41,42]. Nevertheless, there are other studies that show different values than what we reported. Unfortunately, there is no consensus in the literature. While the ACL is certainly one of the most significant and well described ligaments, many other ligaments are critical to joint stability throughout the human body. Moreover, surgery for ligament reconstruction is common and standard of care in hand surgery, foot and ankle surgery, orthopedic trauma surgery and even spine surgery [58-60]. In general, ligaments of joints smaller than the knee are reconstructed using Ethicon, fibre wire, Ethibond sutures or mersilene tape. Although our 3D-printed materials were not fit for the high biomechanical demands of the knee, we believe the characteristics of these biomaterials, such as freedom of printable complex geometries, potential resorptive capacities and biomechanical strength, may prove to be a dynamic and promising alternative in many other smaller and less biomechanically demanding ligament reconstruction scenarios.

In this study, we determined the effect of raster angle on the mechanical properties of a number of stiff and flexible materials as candidates for mechanically functional ACL scaffolds. Whilst there was no single material that matched literature values for the native ACL, PLA and PETG had comparable ultimate tensile strengths and Lay FOMM 60 had the closest percentage strain at failure. A combination of PLA/PETG and Lay FOMM 60 to co-print biomimetic ACL constructs should be attempted in future studies. The mechanical properties of PLA and PETG were altered by the raster angle, which suggests that they may be used to replicate the anisotropic properties of the ACL. Co-printing with Lay FOMM 60 may increase the construct's strain at failure and yield a more functional structure. To overcome the described shortcomings, a robust cadaveric study of the ACL should be implemented. Future studies into co-printing of these materials into composite structures and introducing porosity will hopefully enable the production of a more mechanically appropriate scaffold. Then, a comparison of several 
samples of cadaveric ACL to the real values of co-printed constructs should be performed. In addition, in-depth in vitro and in vivo studies with the use of scaffolds should be conducted once appropriate materials are identified with optimal mechanics and favourable biocompatibility.

Supplementary Materials: The following are available online at http://www.mdpi.com/2072-666X/11/9/846/s1, Table S1: Results of one-way ANOVA Tukey's multiple comparison test comparing the mechanical properties of flexible Significance values of apparent modulus, 5\% strain, and 20\% strain for flexible specimens having a raster angle of $0^{\circ}$, Table S2: Results of one-way ANOVA Tukey's multiple comparison test comparing the mechanical properties of flexible specimens with a raster angle of $45^{\circ}$, Table S3: Results of one-way ANOVA Tukey's multiple comparison test comparing the mechanical properties of flexible specimens with a raster angle of $90^{\circ}$.

Author Contributions: Conceptualization M.E.C., M.H.W., P.A.M. and D.H.R.; methodology, A.A.P., J.-G.L., M.E.C., L.B. and T.S.; 3D printing and G-Code optimization; M.E.C. and A.A.P.; formal analysis, A.A.P. and J.-G.L.; writing-original draft preparation, A.A.P. and J.-G.L.; writing-review and editing, all authors contributed. All authors have read and agreed to the published version of the manuscript.

Funding: This research was supported by start-up funds from the Research Institute of McGill University Health Centre to D.H.R. JG.L. and M.E.C. were supported by a studentship and postdoctoral fellowship awards from Reseau de Recherche en Sante Buccodentaire et Osseuse (RSBO).

Conflicts of Interest: The authors declare no conflict of interest.

\section{References}

1. Risberg, M.A.; Lewek, M.; Snyder-Mackler, L. A systematic review of evidence for anterior cruciate ligament rehabilitation: How much and what type? Phys. Ther. Sport 2004, 5, 125-145. [CrossRef]

2. Butler, D.L.; Noyes, F.R.; Grood, E.S. Ligamentous restraints to anterior-posterior drawer in the human knee. A biomechanical study. J. Bone Jt. Surg. Am. Vol. 1980, 62, 259-270. [CrossRef]

3. Kiapour, A.M.; Murray, M.M. Basic science of anterior cruciate ligament injury and repair. Bone Jt. Res. 2014, 3, 20-31. [CrossRef] [PubMed]

4. Gallucci, J. Soccer Injury Prevention and Treatment: A Guide to Optimal Performance for Players, Parents, and Coaches; Demos Medical Publishing: New York, NY, USA, 2014; p. 201.

5. Marieswaran, M.; Jain, I.; Garg, B.; Sharma, V.; Kalyanasundaram, D. A review on biomechanics of anterior cruciate ligament and materials for reconstruction. Appl. Bionics Biomech. 2018, 2018, 4657824. [CrossRef] [PubMed]

6. Markatos, K.; Kaseta, M.K.; Lallos, S.N.; Korres, D.S.; Efstathopoulos, N. The anatomy of the acl and its importance in acl reconstruction. Eur. J. Orthop. Surg. Traumatol. Orthop. Traumatol. 2013, 23, 747-752. [CrossRef] [PubMed]

7. Arnoczky, S.P. Anatomy of the anterior cruciate ligament. Clin. Orthop. Relat. Res. 1983, 172, $19-25$. [CrossRef]

8. Mommersteeg, T.J.; Kooloos, J.G.; Blankevoort, L.; Kauer, J.M.; Huiskes, R.; Roeling, F.Q. The fibre bundle anatomy of human cruciate ligaments. J. Anat. 1995, 187, 461-471.

9. Welsh, R.P. Knee joint structure and function. Clin. Orthop. Relat. Res. 1980, 147, 7-14. [CrossRef]

10. Amis, A.A.; Dawkins, G.P. Functional anatomy of the anterior cruciate ligament. Fibre bundle actions related to ligament replacements and injuries. J. Bone Jt. Surg. Br. Vol. 1991, 73, 260-267. [CrossRef]

11. Norwood, L.A.; Cross, M.J. Anterior cruciate ligament: Functional anatomy of its bundles in rotatory instabilities. Am. J. Sports Med. 1979, 7, 23-26. [CrossRef]

12. Blackburn, T.A.; Craig, E. Knee anatomy: A brief review. Phys. Ther. 1980, 60, 1556-1560. [CrossRef]

13. Huebner, P.; Warren, P.B.; Chester, D.; Spang, J.T.; Brown, A.C.; Fisher, M.B.; Shirwaiker, R.A. Mechanical properties of tissue formed in vivo are affected by $3 \mathrm{~d}$-bioplotted scaffold microarchitecture and correlate with ecm collagen fiber alignment. Connect. Tissue Res. 2020, 61, 190-204. [CrossRef]

14. Kwansa, A.L.; Empson, Y.M.; Ekwueme, E.C.; Walters, V.I.; Freeman, J.W.; Laurencin, C.T. Novel matrix based anterior cruciate ligament (acl) regeneration. Soft Matter 2010, 6, 5016. [CrossRef]

15. Śmigielski, R.; Zdanowicz, U.; Drwięga, M.; Ciszek, B.; Ciszkowska-Łysoń, B.; Siebold, R. Ribbon like appearance of the midsubstance fibres of the anterior cruciate ligament close to its femoral insertion site: A cadaveric study including 111 knees. Knee Surg. Sports Traumatol. Arthrosc. 2015, 23, 3143-3150. [CrossRef] 
16. Korenczuk, C.E.; Votava, L.E.; Dhume, R.Y.; Kizilski, S.B.; Brown, G.E.; Narain, R.; Barocas, V.H. Isotropic failure criteria are not appropriate for anisotropic fibrous biological tissues. J. Biomech. Eng. 2017, 139, 0710081-07100810.

17. Friel, N.A.; Chu, C.R. The role of acl injury in the development of posttraumatic knee osteoarthritis. Clin. Sports Med. 2013, 32, 1-12. [CrossRef]

18. Mahapatra, P.; Horriat, S.; Anand, B.S. Anterior cruciate ligament repair-Past, present and future. J. Exp. Orthop. 2018, 5, 1-10. [CrossRef] [PubMed]

19. Mastrangelo, A.N.; Magarian, E.M.; Palmer, M.P.; Vavken, P.; Murray, M.M. The effect of skeletal maturity on the regenerative function of intrinsic acl cells. J. Orthop. Res. 2010, 28, 644-651. [CrossRef] [PubMed]

20. Laurent, C.D.; Liu, X.; De Isla, N.; Wang, X.; Rahouadj, R. Defining a scaffold for ligament tissue engineering: What has been done, and what still needs to be done. J. Cell. Immunother. 2018, 4, 4-9. [CrossRef]

21. Frank, C.B. Ligament structure, physiology and function. J. Musculoskelet. Neuronal Interact. 2004, 4, $199-201$.

22. Crawford, S.N.; Waterman, B.R.; Lubowitz, J.H. Long-term failure of anterior cruciate ligament reconstruction. Arthrosc. J. Arthrosc. Relat. Surg. 2013, 29, 1566-1571. [CrossRef]

23. Diegel, O. 10.02-Additive manufacturing: An overview. In Comprehensive Materials Processing; Elsevier Ltd.: Oxford, UK, 2014.

24. Jiménez, M.; Romero, L.; Domínguez, I.A.; Espinosa, M.D.M.; Domínguez, M. Additive manufacturing technologies: An overview about 3d printing methods and future prospects. Complexity 2019, 2019, 1-30. [CrossRef]

25. Ahangar, P.; Cooke, M.E.; Weber, M.H.; Rosenzweig, D.H. Current biomedical applications of 3d printing and additive manufacturing. Appl Sci. 2019, 9, 1713. [CrossRef]

26. Haglund, L.; Ahangar, P.; Rosenzweig, D.H. Advancements in $3 d$ printed scaffolds to mimic matrix complexities for musculoskeletal repair. Curr. Opin. Biomed. Eng. 2019, 10, 142-148. [CrossRef]

27. Gleadall, A.; Visscher, D.; Yang, J.; Thomas, D.; Segal, J. Review of additive manufactured tissue engineering scaffolds: Relationship between geometry and performance. Burns Trauma 2018, 6, 19. [CrossRef] [PubMed]

28. O'Brien, F.J. Biomaterials \& scaffolds for tissue engineering. Mater. Today 2011, 14, 88-95.

29. Ratcliffe, A.; Butler, D.L.; Dyment, N.A.; Cagle, P.J.; Proctor, C.S.; Ratcliffe, S.S.; Flatow, E.L. Scaffolds for tendon and ligament repair and regeneration. Ann. Biomed. Eng. Soc. 2015, 43, 819-831. [CrossRef]

30. Lim, W.L.; Liau, L.L.; Ng, M.H.; Chowdhury, S.R.; Law, J.X. Current progress in tendon and ligament tissue engineering. Tissue Eng. Regen. Med. 2019, 16, 549-571. [CrossRef]

31. Rosenzweig, D.H.; Carelli, E.; Steffen, T.; Jarzem, P.; Haglund, L. 3d-printed abs and pla scaffolds for cartilage and nucleus pulposus tissue regeneration. Int. J. Mol. Sci. 2015, 16, 15118-15135. [CrossRef]

32. Fairag, R.; Rosenzweig, D.H.; Ramirez-Garcialuna, J.L.; Weber, M.H.; Haglund, L. Three-dimensional printed polylactic acid scaffolds promote bone-like matrix deposition in vitro. ACS Appl. Mater. Interfaces 2019, 11, 15306-15315. [CrossRef]

33. Alaribe, F.N.; Manoto, S.L.; Motaung, S.C.K.M. Scaffolds from biomaterials: Advantages and limitations in bone and tissue engineering. Biologia 2016, 71, 353-366. [CrossRef]

34. Eltom, A.; Zhong, G.; Muhammad, A. Scaffold techniques and designs in tissue engineering functions and purposes: A review. Adv. Mater. Sci. Eng. 2019, 2019, 1-13. [CrossRef]

35. Ahangar, P.; Akoury, E.; Luna, A.S.R.G.; Nour, A.; Weber, M.H.; Rosenzweig, D.H. Nanoporous 3d-printed scaffolds for local doxorubicin delivery in bone metastases secondary to prostate cancer. Materials 2018, 11, 1485. [CrossRef]

36. TDS. NinjaFlex; Fenner Inc.: Manheim, PA, USA, 2016; Available online: https://ninjatek.com/wp-content/ uploads/2019/10/NinjaFlex-TDS.pdf (accessed on 9 March 2020).

37. TDS. NinjaFlex SemiFlex; Fenner Inc.: Manheim, PA, USA, 2016; Available online: https://www.3dmensionals. de/media/pdf/cf/33/7e/SemiFlex-TDS.pdf (accessed on 9 March 2020).

38. TDS. FlexiFil; Formfutura BV: Nijmegan, The Netherlands. Available online: https://gzhls.at/blob/ldb/0/4/1/7/ 4ddb1d5c17caa564a350f22266fb04ae1b39.pdf (accessed on 9 March 2020).

39. TDS. PLA; SD3D Printing: San Diego, CA, USA. Available online: https://www.sd3d.com/wp-content/ uploads/2017/06/MaterialTDS-PLA_01.pdf (accessed on 9 March 2020).

40. TDS. PETG; SD3D Printing: San Diego, CA, USA. Available online: https://www.sd3d.com/wp-content/ uploads/2017/06/MaterialTDS-PETG_01.pdf (accessed on 9 March 2020). 
41. Takeda, Y.; Xerogeanes, J.W.; Livesay, G.A.; Fu, F.H.; Woo, S.L.Y. Biomechanical function of the human anterior cruciate ligament. J. Arthrosc. Relat. Surg. 1994, 10, 140-147. [CrossRef]

42. Chandrashekar, N.; Mansouri, H.; Slauterbeck, J.; Hashemi, J. Sex-based differences in the tensile properties of the human anterior cruciate ligament. J. Biomech. 2006, 39, 2943-2950. [CrossRef]

43. Parrado-Agudelo, J.Z.; Narváez-Tovar, C. Mechanical characterization of polylactic acid, polycaprolactone and lay-fomm 40 parts manufactured by fused deposition modeling, as a function of the printing parameters. ITECKNE 2019, 16, 25-31. [CrossRef]

44. Fleming, B.C.; Beynnon, B.D. In vivo measurement of ligament/tendon strains and forces: A review. Ann. Biomed. Eng. 2004, 32, 318-328. [CrossRef]

45. Beynnon, B.D.; Fleming, B.C. Anterior cruciate ligament strain in-vivo: A review of previous work. J. Biomech. 1998, 31, 519-525. [CrossRef]

46. Sheehan, F.T.; Rebmann, A. Non-invasive, in vivo measures of anterior cruciate ligament strains. Trans. in Orthop. Res. Soc. 2003, 28, 264.

47. Butler, D.L.; Guan, Y.; Kay, M.D.; Cummings, J.F.; Feder, S.M.; Levy, M.S. Location-dependent variations in the material properties of the anterior cruciate ligament. J. Biomech. 1992, 25, 511-518. [CrossRef]

48. Bružauskaitè, I.; Bironaitè, D.; Bagdonas, E.; Bernotienè, E. Scaffolds and cells for tissue regeneration: Different scaffold pore sizes-Different cell effects. Cytotechnol. Inc. Methods Cell Sci. Int. J.Cell Cult. Biotechnol. 2016, 68, 355-369.

49. Akoury, E.; Weber, M.H.; Rosenzweig, D.H. 3d-printed nanoporous scaffolds impregnated with zoledronate for the treatment of spinal bone metastases. MRS Adv. 2019, 4, 1245-1251. [CrossRef]

50. Wang, S.; Zhong, S.; Lim, C.T.; Nie, H. Effects of fiber alignment on stem cells-fibrous scaffold interactions. J. Mater. Chem. B 2015, 3, 3358-3366. [CrossRef]

51. Brown, R.A.; Prajapati, R.; McGrouther, D.A.; Yannas, I.V.; Eastwood, M. Tensional homeostasis in dermal fibroblasts: Mechanical responses to mechanical loading in three-dimensional substrates. J. Cell. Physiol. 1998, 175, 323-332. [CrossRef]

52. Wang, J.H.C.; Jia, F.; Gilbert, T.W.; Woo, S.L.Y. Cell orientation determines the alignment of cell-produced collagenous matrix. J. Biomech. 2003, 36, 97-102. [CrossRef]

53. Tanikella, N.G.; Wittbrodt, B.; Pearce, J.M. Tensile strength of commercial polymer materials for fused filament fabrication 3d printing. Addit. Manuf. 2017, 15, 40-47. [CrossRef]

54. Perego, G.; Cella, G.D.; Bastioli, C. Effect of molecular weight and crystallinity on poly(lactic acid) mechanical properties. J. Appl. Polym. Sci. 1996, 59, 37-43. [CrossRef]

55. Wittbrodt, B.; Pearce, J.M. The effects of pla color on material properties of 3-d printed components. Addit. Manuf. 2015, 8, 110-116. [CrossRef]

56. Wimpenny, D.I.; Pandey, P.M.; Kumar, L.J. Advances in $3 d$ Printing $\mathcal{E}$ Additive Manufacturing Technologies; Springer: Singapore, 2016.

57. Balani, K.; Verma, V.; Agarwal, A.; Narayan, R. Biosurfaces: A Materials Science and Engineering Perspective; John Wiley \& Sons, Inc.: Hoboken, NJ, USA, 2015; p. 338.

58. Mar, D.E.; Clary, S.J.; Burton, D.C.; McIff, T.E. Biomechanics of prophylactic tethering for proximal junctional kyphosis: Characterization of spinous process tether pretensioning and pull-out force. Spine Deform. 2019, 7, 191-196. [CrossRef]

59. Petersen, W.; Rembitzki, I.V.; Koppenburg, A.G.; Ellermann, A.; Liebau, C.; Brüggemann, G.P.; Best, R. Treatment of acute ankle ligament injuries: A systematic review. Arch. Orthop. Trauma Surg. 2013, 133, 1129-1141. [CrossRef]

60. Andersson, J.K.; Rööser, B.; Karlsson, J. Level of evidence in wrist ligament repair and reconstruction research: A systematic review. J. Exp. Orthop. 2018, 5, 1-8. [CrossRef] [PubMed]

(C) 2020 by the authors. Licensee MDPI, Basel, Switzerland. This article is an open access article distributed under the terms and conditions of the Creative Commons Attribution (CC BY) license (http://creativecommons.org/licenses/by/4.0/). 